\title{
ON THE AMPLE CONE OF AN AMPLE HYPERSURFACE *
}

\section{BALÁZS SZENDRÖI ${ }^{\dagger}$}

For a smooth ample hypersurface $D$ of dimension at least 3 in a smooth projective variety $X$, the restriction map $r: \operatorname{Pic}(X) \rightarrow \operatorname{Pic}(D)$ is an isomorphism by weak Lefschetz. In a recent paper [3], Hassett, Lin and Wang gave an example of a pair $D \subset X$ with $\operatorname{dim} D=3$ such that the cone of ample divisor classes of $D$ is strictly larger than the image under $r$ of the cone of ample divisor classes of $X$; in other words, the weak Lefschetz principle can fail for ample cones.

The purpose of this note is to point out that not only is the ample cone of a hypersurface $D$ often larger than the ample cone of the ambient space $X$, in general it cannot even be computed from birational models of $X$. This contrasts with the conjecture of [2] (already disproved for semi-ample hypersurfaces in [5]), which predicted that such a description is possible. Note that in the special case studied in [3], the ample cone is the union of ample cones of birational models of $X$ (Remark 2.3). However, a slight generalization of the example gives hypersurfaces with ample cone larger than the union of ample cones of all birational models of the ambient space (Corollary 3.1).

For a projective variety $Y$, denote by $\mathcal{N}(Y) \subset \operatorname{Pic}_{\mathbb{R}}(Y)$ the cone of nef divisor classes; this is the closure of the ample cone and also the dual of the Mori cone by Kleiman's criterion. Facets (codimension-one faces) of $\mathcal{N}(Y)$ correspond in favourable cases to primitive (non-factorizable) contraction morphisms on $Y$.

1. The ambient space. I use the notation of [3]. Let $\varphi: X \rightarrow \mathbb{P}^{4}$ be the blowup of two distinct points $p_{1}, p_{2} \in \mathbb{P}^{4}$. The line $p_{1} p_{2}=l^{\prime} \subset \mathbb{P}^{4}$ has proper transform $l \subset X$. The Picard group $\operatorname{Pic}(X)=\left\langle H, E_{1}, E_{2}\right\rangle$ is generated by the classes of exceptional divisors $E_{1}, E_{2}$ of $\varphi$ and the proper transform $H$ of a general hyperplane section of $\mathbb{P}^{4}$ through $p_{1}, p_{2}$. Let $\mathcal{B}, \mathcal{C}$ be the cones in $\operatorname{Pic}_{\mathbb{R}}(X)$ spanned by the rays $H+E_{1}, H+E_{2}, H+E_{1}+E_{2}$ and $H, H+E_{1}, H+E_{2}$ respectively.

Proposition 1.1.

(i) The nef cone of $X$ is $\mathcal{N}(X)=\mathcal{B}$; the three facets of the cone correspond to the contractions of $E_{i}$, and to a small contraction $\psi: X \rightarrow \bar{X}$ contracting the single extremal curve $l \cong \mathbb{P}^{1}$ with $K_{X} \cdot l=1$.

(ii) The antiflip $\psi^{+}: X^{+} \rightarrow \bar{X}$ of $\psi$ exists. The proper transform $E_{i}^{+}$of $E_{i}$ is isomorphic to $\mathbb{P}^{3}$ blown up in a point; $E_{1}^{+} \cap E_{2}^{+} \cong \mathbb{P}^{2}$ is the exceptional divisor of both blowups and also the small exceptional locus of $\psi^{+}$.

(iii) Let $\alpha: X^{+} \rightarrow X$ be the flip and identify the Picard groups of $X$ and $X^{+}$ by the map $\alpha_{*}: \operatorname{Pic}\left(X^{+}\right) \stackrel{\sim}{\longrightarrow} \operatorname{Pic}(X)$ induced by proper transform on divisors composed with the isomorphisms between the Picard groups of $X, X^{+}$ and their class groups. Then $\mathcal{N}\left(X^{+}\right)=\mathcal{C}$. The three facets of the cone $\mathcal{C}$ correspond to the contraction $\psi^{+}$and two different $\mathbb{P}^{1}$-bundle structures $\pi_{i}: X^{+} \rightarrow Z$. The image $Z$ is the blowup of $\mathbb{P}^{3}$ in one point. The fibres $l_{i}$ of $\pi_{i}$ are generically the lines of the original $\mathbb{P}^{4}$ through the point $p_{i}$. On $E_{i}^{+}$, $\pi_{i}$ is the identity; on $E_{3-i}^{+}$it is the unique projection to $\mathbb{P}^{2}$.

${ }^{*}$ Received May 9, 2002; accepted for publication November 15, 2002.

† Mathematics Institute, University of Warwick, Coventry CV4 7AL, United Kingdom and Alfréd Rényi Institute of Mathematics, Hungarian Academy of Sciences (balazs@maths.warwick.ac.uk). 
Proof. Once the statement is formulated, the proof becomes an elementary exercise; my personal favourite is toric geometry, which provides a clean and uniform proof. In the lattice $N=\mathbb{Z}^{4}$, the fan spanned by

$$
v_{0}=(-1,-1,-1,-1), v_{3}=(1,0,0,0), \ldots, v_{6}=(0,0,0,1)
$$

describes $\mathbb{P}^{4}$. Barycentric subdivision of the cones $v_{0} v_{3} v_{4} v_{5}$ and $v_{0} v_{3} v_{4} v_{6}$ by the vertices

$$
v_{1}=(0,0,0,-1), v_{2}=(0,0,-1,0)
$$

leads to $X$. In this language, $E_{i}$ are the torus-invariant divisors corresponding to the rays $v_{i}$ and $l$ is the torus orbit corresponding to the facet $v_{0} v_{3} v_{4}$. A short check using toric Mori theory [4] shows that there are three primitive contractions on $X$ : the contractions of $E_{i}$ and a contraction $\psi$ with one-dimensional exceptional locus $l$. The extremal curves define three inequalities on ample classes which gives $\mathcal{N}(X)=\mathcal{B}$.

The fan of $X^{+}$is obtained by replacing the cones $v_{0} v_{1} v_{3} v_{4}$ and $v_{0} v_{2} v_{3} v_{4}$ by the cones $v_{0} v_{1} v_{2} v_{3}, v_{0} v_{1} v_{2} v_{4}$ and $v_{1} v_{2} v_{3} v_{4}$. The new codimension-two face $v_{1} v_{2}$ represents the small exceptional locus $E_{1}^{+} \cap E_{2}^{+} \cong \mathbb{P}^{2}$. There are three primitive contractions again; the two $\mathbb{P}^{1}$-bundle structures $\pi_{i}$ come from projecting the whole fan along the vector $v_{i}$ for $i=1,2$. The tasks of drawing the pictures and filling in the details are left to the reader.

2. The hypersurface. Let now $D^{\prime} \subset \mathbb{P}^{4}$ be a smooth hypersurface of degree $d>2$ containing $p_{1}$ and $p_{2}$; assume for simplicity that the line $l^{\prime}$ is either contained in $D^{\prime}$ or is at most simply tangent to $D^{\prime}$ at $p_{i}$ for $i=1,2$ while meeting it transversally elsewhere. Let $D \subset X$ be the proper transform of $D^{\prime}$. A simple computation shows that $D$ is ample in $X$; hence by the Lefschetz principle, the Picard groups of $X$ and $D$ can be identified by the restriction isomorphism $r: \operatorname{Pic}(X) \rightarrow \operatorname{Pic}(D)$.

Proposition 2.1.

(i) If $l^{\prime} \subset D^{\prime}$, then

$$
\mathcal{N}(D)=\mathcal{B}
$$

(ii) Assume that $D^{\prime}$ does not contain $l^{\prime}$, but for both indices $i=1,2$ one of the following holds:

a. $D^{\prime}$ contains a line through $p_{i}$, or

b. the line $l^{\prime}$ is tangent to $D^{\prime}$ at $p_{3-i}$.

Then

$$
\mathcal{N}(D)=\mathcal{B} \cup \mathcal{C}
$$

(iii) In all other cases, there is a strict inclusion

$$
\mathcal{N}(D) \supset \mathcal{B} \cup \mathcal{C}
$$

Proof. $\mathcal{N}(D) \supset \mathcal{B}$ is clear as a restriction of an ample class from $X$ to $D$ is also ample. Part (i) follows immediately from Proposition 1.1(i), since every face of the nef cone of $X$ corresponds to a contraction with $D$ containing at least one fibre.

To prove (ii), let $\bar{D} \subset \bar{X}, D^{+} \subset X^{+}$be the proper transforms of $D$ in $\bar{X}$ and $X^{+}$. As $D$ meets $l$ in a finite number of points, the map $\left.\psi\right|_{D}: D \rightarrow \bar{D}$ is finite and 

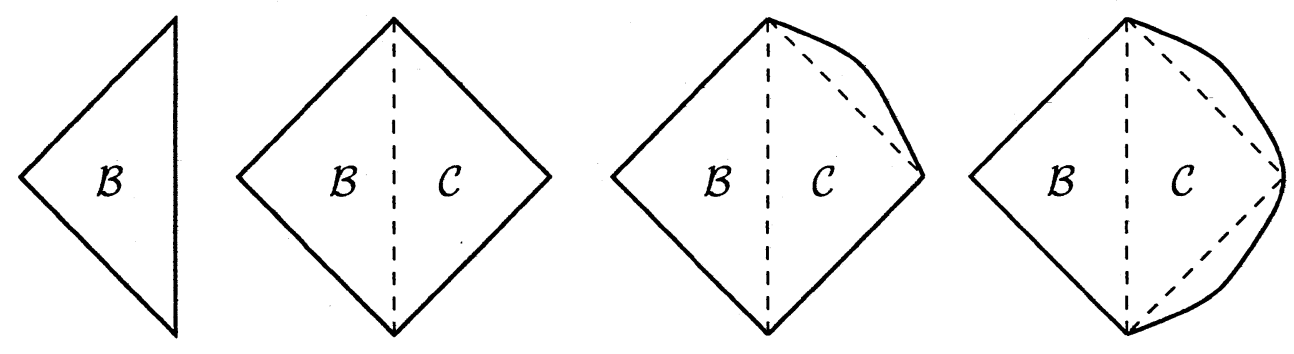

FIG. 2.1. Possibilities for the shape of $\mathcal{N}(D)$

birational and there is a Cartesian diagram

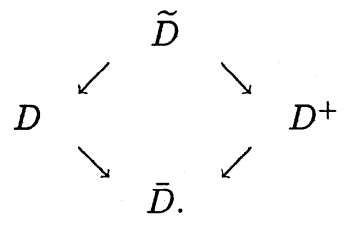

By the conditions put on $D^{\prime}$, the map $\sigma: \widetilde{D} \rightarrow D$ is the blowup of the intersection points of $l$ and $D$; the map $\tau: \widetilde{D} \rightarrow D^{+}$is again finite and birational. Write $\beta: D^{+} \rightarrow D$ for the composition $\sigma \circ \tau^{-1}$.

Take any ample divisor class $A^{+}$on $X^{+}$. Part (ii) will follow if I can show that the divisor class $r\left(\alpha_{*} A^{+}\right)$is ample on $D$. However, using the fact that $\tau$ is finite birational,

$$
r\left(\alpha_{*} A^{+}\right)=\beta_{*} r^{+}\left(A^{+}\right)=\sigma_{*} \tau^{*} r^{+}\left(A^{+}\right)
$$

where $r^{+}: \operatorname{Pic}\left(Y^{+}\right) \rightarrow \operatorname{Pic}\left(X^{+}\right)$is the restriction map. As $A^{+}$is ample on $Y^{+}$, $r^{+}\left(A^{+}\right)$is an ample Cartier divisor class on $X^{+}$and hence $\tau^{*} r^{+}\left(A^{+}\right)$is ample on $\widetilde{D}$. Now the ampleness of $r\left(\alpha_{*} A^{+}\right)$on $D$ follows by applying Lemma 2.2 to the map $\sigma: \widetilde{D} \rightarrow D$.

To prove (iii), take $A=\pi_{i}^{*} B$ where $B$ is ample on $Z ; A$ is a nef but non-ample divisor class in the boundary of the cone $\mathcal{N}\left(X^{+}\right)$. If none of the special circumstances of (i)-(ii) happen, then $D^{+}$does not contain any fibre of $\pi_{i}$, so $\pi_{i}$ restricts to $D^{+}$as a finite morphism. Hence the restriction of $A$ to $D^{+}$is ample. The argument of (ii) now shows that $r\left(\alpha_{*} A\right)$ is ample on $D$ and as ampleness is an open condition, (iii) follows.

LEMMA 2.2. Let $Y$ be a smooth threefold, $f: \widetilde{Y} \rightarrow Y$ the blowup of a closed point. If $A$ is an ample divisor class on $\tilde{Y}$, then its proper transform $B=f_{*} A$ is ample on $Y$.

Proof. Let $E$ be the exceptional divisor; then $f^{*} B=A+\lambda E$ with a non-negative integer $\lambda$. If $C$ is a curve in $Y$ with proper transform $\widetilde{C}$ in $\widetilde{Y}$, then

$$
B \cdot C=f^{*} B \cdot \widetilde{C}=A \cdot \widetilde{C}+\lambda E \cdot \widetilde{C}>0
$$


as $A \cdot \widetilde{C}>0$ and $E \cdot \widetilde{C} \geq 0$ since $\widetilde{C}$ is not contained in $E$. If $S$ is any surface in $X$ with proper transform $\widetilde{S}$ then

$$
B^{2} \cdot S=(A+\lambda E)^{2} \widetilde{S}=A \cdot(A+\lambda E) \cdot \widetilde{S} \geq A^{2} \cdot \widetilde{S}>0 .
$$

The second equality holds since $(A+\lambda E) \cdot E \cdot \widetilde{S}=\left.f^{*} B \cdot \widetilde{S}\right|_{E}=0$ by the projection formula, as $E$ and $\widetilde{S}$ intersect in a curve contracted by $f$; the first inequality uses $A \cdot E \cdot \widetilde{S}=\left.A \cdot \widetilde{S}\right|_{E} \geq 0$. Finally in same way

$$
B^{3}=(A+\lambda E)^{3} \geq(A+\lambda E)^{2} \cdot A=(A+\lambda E) \cdot A^{2} \geq A^{3}>0 .
$$

Hence by the Nakai-Moishezon criterion, $B$ is ample.

REMARK 2.3. Note that in the special case of smooth cubics studied in [3], the first condition of Proposition 2.1(ii) is always satisfied: a smooth cubic threefold is covered by lines. Hence either $l^{\prime} \subset D^{\prime}$ and $\mathcal{N}(D)=\mathcal{B}$, or $l^{\prime} \not D^{\prime}$ and $\mathcal{N}(D)=\mathcal{B} \cup \mathcal{C}$. [3, Assumptions 2.5] fixes the type of the primitive contractions on $D$ induced by $\pi_{i}$ : if $D^{\prime}$ contains finitely many lines through $p_{i}$ and $l^{\prime}$ is not tangent to $D^{\prime}$ at $p_{i}$, then (the Stein factorization of $)\left.\pi_{i}\right|_{D}$ is a small flopping contraction $\left(K_{D} \cdot l_{i}=0\right)$; otherwise it is divisorial.

3. Good hypersurfaces. In the original example of [3], as well as in all cases where the conditions of Proposition 2.1(i)-(ii) are satisfied, the ample cone of the hypersurface $D$ has an explicit description in terms of nef cones of models of its ambient space; $D$ is good in the following sense:

Good hypersurface: Call a hypersurface $D \subset X$ good, if the nef cone $\mathcal{N}(D)$ is a union of cones $r\left(\alpha_{i *} \mathcal{N}\left(X_{i}\right)\right.$ ), where $\alpha_{i}: X_{i} \rightarrow X$ are certain birational models of $X$ isomorphic to $X$ in codimension one.

Anticanonical hypersurfaces in certain toric varieties were conjectured to be good by Cox and Katz [2, Conjecture 6.2.8]. By [5], this conjecture can fail if $D$ is only semi-ample. Alas, an ample but non-anticanonical $D \subset X$ can also fail to be good.

Corollary 3.1. Take any smooth degree $d>3$ hypersurface $D^{\prime}$ in $\mathbb{P}^{4}$ with no lines through $p_{i}$ and with $l^{\prime}$ not tangent at $p_{i}$ for both $i=1,2$ and let $D$ be its proper transform in $X$ as before. Then $D \subset X$ is not good.

Proof. By Proposition 2.1(iii), $\mathcal{N}(D)$ is strictly larger than $\mathcal{B} \cup \mathcal{C}$. On the other hand, $X$ has no further birational models which are isomorphic in codimension one. For if $\alpha_{i}: X_{i} \rightarrow X$ is any such model, then $\alpha_{i *} A$ is a moving divisor class for $A$ ample on $X_{i}$. However, from the explicit description of Proposition 1.1 it is clear that the moving cone of $X$ is the interior of $\mathcal{B} \cup \mathcal{C}$. So $X_{i}$ is isomorphic to $X$ or $X^{+}$.

Note that a 3-dimensional ample and anticanonical hypersurface is always good: if $D \in\left|-K_{X}\right|$ is ample, $r(\mathcal{N}(X))=\mathcal{N}(D)$ by Kollár's result [1]. To the best of my knowledge, the analogous result is not known if $\operatorname{deg}(D)>3$.

Acknowledgements. I thank Brendan Hassett for bringing [3] to my attention, Massimiliano Mella for pointing out an error in the first version and Miles Reid for help with Lemma 2.2. 


\section{REFERENCES}

[1] C. BorceA, Homogeneous vector bundles and families of Calabi-Yau threefolds II, with an Appendix by János Kollár, in: Proc. Sympos. in Pure Math. 52 (ed. E. Bedford et al), Part 2. 83-91, AMS, Providence, RI, 1991.

[2] D.A. Cox And S. KATZ, Mirror symmetry and algebraic geometry, Mathematical Surveys and Monographs 68, American Mathematical Society, Providence, 1999.

[3] B. HASSETT, H.-W. LIN AND C.-L. WANG, The weak Lefschetz principle is false for ample cones, Asian J. Math., 6 (2002), pp. 95-99.

[4] M. ReID, Decomposition of toric morphisms, in: Algebra and Geometry, papers dedicated to I.R. Shafarevich on the occasion of his 60th birthday (eds. M. Artin and J. Tate), vol. II. 395-418, Progr. Math. 36, Birkhauser, Boston, Basel, Stuttgart, 1983.

[5] B. SzendröI, On a conjecture of Cox and Katz, Math. Zeitschrift, 240 (2002), pp. 233-241. 
B. SZENDRŐI 